Correction

\title{
Correction: Lin, Y., et al. Road Extraction from Very-High-Resolution Remote Sensing Images via a Nested SE-Deeplab Model. Remote Sens. 2020, 12, 2985
}

\author{
Yeneng Lin ${ }^{1}\left(\mathbb{D}\right.$, Dongyun $\mathrm{Xu}^{1}{ }^{1}$, Nan Wang ${ }^{1}$, Zhou Shi $\left.{ }^{1} \mathbb{(}\right)$ and Qiuxiao Chen ${ }^{2, *} \mathbb{C}$ \\ 1 Institute of Agricultural Remote Sensing and Information Technology Application, College of Environmental \\ and Resource Sciences, Zhejiang University, Hangzhou 310058, China; 21814115@zju.edu.cn (Y.L.); \\ xudongyun@zju.edu.cn (D.X.); wangnanfree@zju.edu.cn (N.W.); shizhou@zju.edu.cn (Z.S.) \\ 2 Department of Regional and Urban Planning, College of Civil Engineering and Architecture, \\ Zhejiang University, Hangzhou 310058, China \\ * Correspondence: chen_qiuxiao@zju.edu.cn
}

check for updates

Citation: Lin, Y.; Xu, D.; Wang, N.; Shi, Z.; Chen, Q. Correction: Lin, Y., et al. Road Extraction from Very-High-Resolution Remote Sensing Images via a Nested SE-Deeplab Model. Remote Sens. 2020, 12, 2985. Remote Sens. 2021, 13, 783. https://doi.org/10.3390/rs13040783

Received: 9 February 2021

Accepted: 16 February 2021

Published: 20 February 2021

Publisher's Note: MDPI stays neutral with regard to jurisdictional claims in published maps and institutional affiliations.

Copyright: (c) 2021 by the authors. Licensee MDPI, Basel, Switzerland. This article is an open access article distributed under the terms and conditions of the Creative Commons Attribution (CC BY) license (https:// creativecommons.org/licenses/by/ $4.0 /)$.
The authors wish to make the following correction to this paper [1]:

\section{Error in Figure/Table}

1. In the original article, there was a mistake in Figure 9. as published. We found a spelling and color marking error in the original Figure 9B, the colors of the red curve and grey curve in the original figure should be exchanged, and 'weightede' should be replaced with 'weighted'. The corrected ${ }^{* *}$ Figure 9.** appears below. We wish to replace

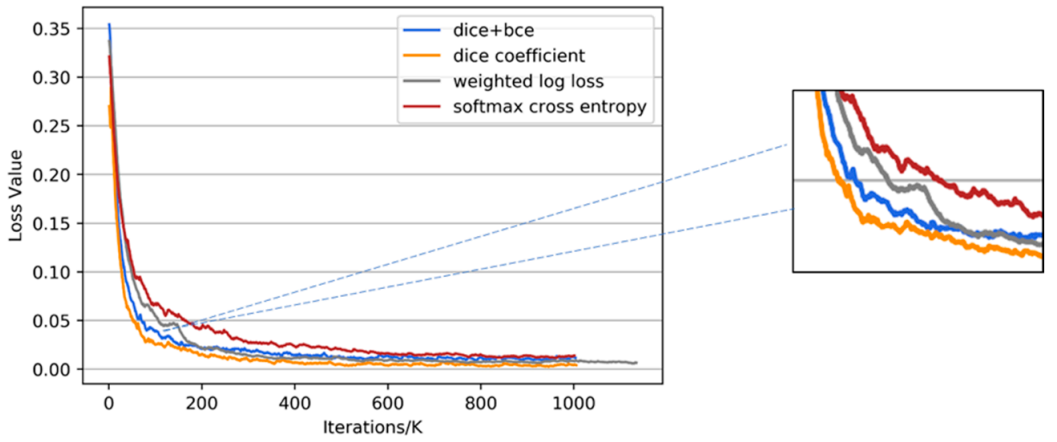

(A)

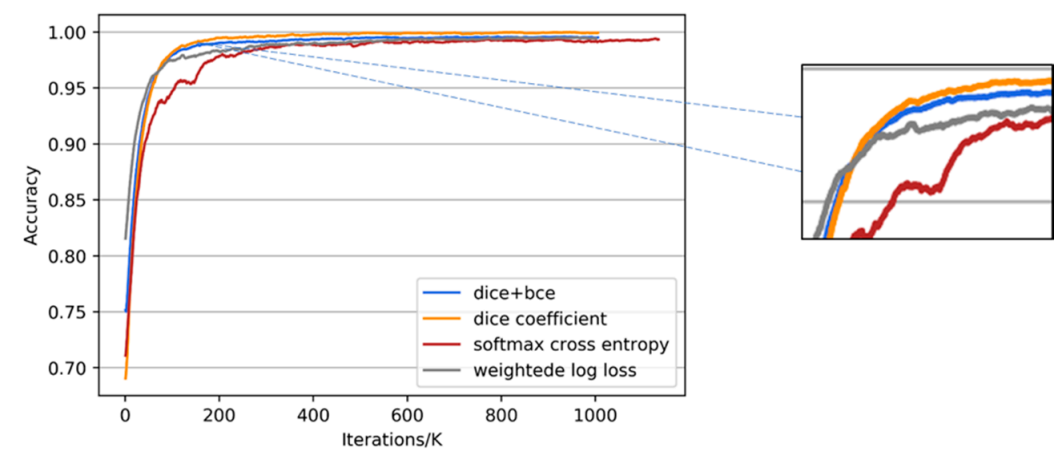

(B)

Figure 9. Progression of loss values (A) and training accuracy (B) for four loss functions used with Nested SE-Deeplab during training. The loss functions are softmax cross entropy (softmax), weighted $\log$ loss, dice coefficient (dice), and dice coefficient added with binary cross entropy (bce). 
with

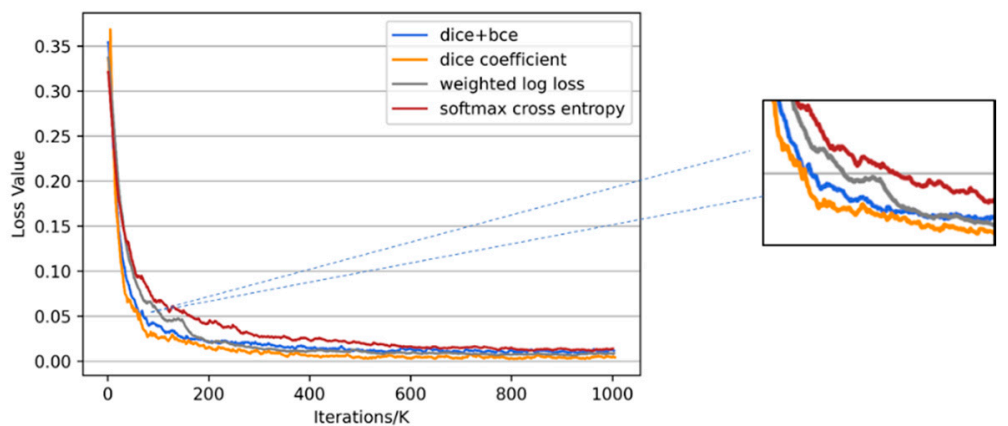

(A)
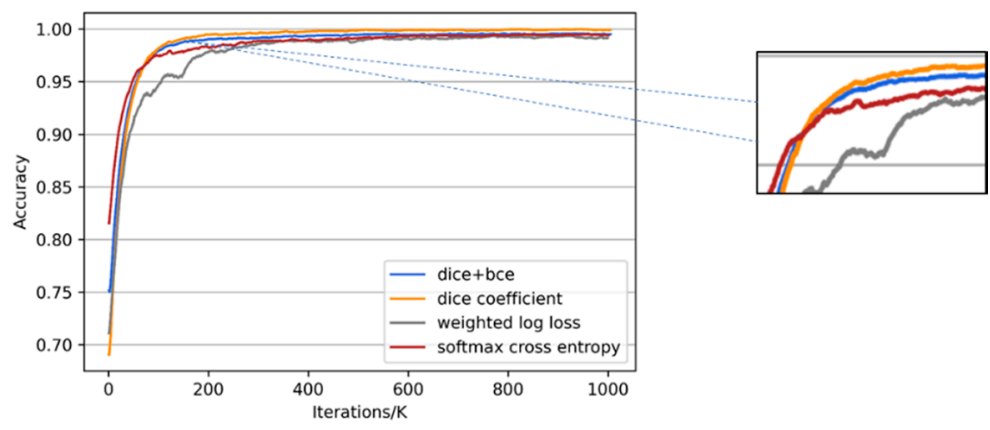

(B)

Figure 9. Progression of loss values (A) and training accuracy (B) for four loss functions used with Nested SE-Deeplab during training. The loss functions are softmax cross entropy (softmax), weighted $\log$ loss, dice coefficient (dice), and dice coefficient added with binary cross entropy (bce).

The authors apologize for any inconvenience caused and state that the scientific conclusions are unaffected. The original article has been updated.

2. In the original article, there was a mistake in Table 3. as published. We are aware that some IoU values in the Table 3 were missed to be updated, so we wish to make this correction. The corrected ${ }^{* *}$ Table $3 .^{* *}$ appears below. We wish to replace

Table 3. Quantitative comparison of three backbone networks for the testing dataset.

\begin{tabular}{ccccc}
\hline Experiment & Methods & Correctness & F1-Score & IoU $^{\mathbf{1}}$ \\
\hline \multirow{3}{*}{ Figure 10a } & ResNet & 0.9100 & 0.9112 & 0.8385 \\
& ResNext & 0.9088 & 0.9161 & 0.8468 \\
& SE-Net & 0.9140 & 0.9167 & 0.8462 \\
\hline \multirow{2}{*}{ Figure 10b } & ResNet & 0.8152 & 0.8274 & 0.7179 \\
& ResNext & 0.8395 & 0.8447 & 0.7312 \\
& SE-Net & 0.8380 & 0.8541 & 0.7454 \\
\hline \multirow{2}{*}{ Figure 10c } & ResNet & 0.7941 & 0.8095 & 0.6957 \\
& ResNext & 0.8120 & 0.8190 & 0.7194 \\
& SE-Net & 0.8270 & 0.8254 & 0.7243 \\
\hline
\end{tabular}

${ }^{1}$ The full name of IoU is Intersection over Union.

with 
Table 3. Quantitative comparison of three backbone networks for the testing dataset.

\begin{tabular}{ccccc}
\hline Experiment & Methods & Correctness & F1-Score & IoU $^{\mathbf{1}}$ \\
\hline \multirow{2}{*}{ Figure 10a } & ResNet & 0.9100 & 0.9112 & 0.8385 \\
& ResNext & 0.9088 & 0.9161 & 0.8455 \\
& SE-Net & 0.9140 & 0.9167 & 0.8462 \\
\hline \multirow{2}{*}{ Figure 10b } & ResNet & 0.8152 & 0.8274 & 0.7056 \\
& ResNext & 0.8395 & 0.8447 & 0.7312 \\
& SE-Net & 0.8380 & 0.8541 & 0.7454 \\
\hline \multirow{2}{*}{ Figure 10c } & ResNet & 0.7941 & 0.8095 & 0.6800 \\
& ResNext & 0.8120 & 0.8190 & 0.6935 \\
& SE-Net & 0.8270 & 0.8254 & 0.7027 \\
\hline
\end{tabular}

${ }^{1}$ The full name of IoU is Intersection over Union.

The authors apologize for any inconvenience caused and state that the scientific conclusions are unaffected. The original article has been updated.

Conflicts of Interest: The authors declare no conflict of interest.

\section{Reference}

1. Lin, Y.; Xu, D.; Wang, N.; Shi, Z.; Chen, Q. Road Extraction from Very-High-Resolution Remote Sensing Images via a Nested SE-Deeplab Model. Remote Sens. 2020, 12, 2985. [CrossRef] 